\title{
Violencia intrafamiliar y de género: Una cuestión educativa
}

\author{
Trabajo de Reflexión y Planteamientos Metodológicos \\ Montserrat Vargas-Vergara ${ }^{13}$
}

\section{Introducción}

A lo largo de este capítulo se va a reflexionar sobre algunas cuestiones importantes relacionadas con el tema de la violencia de género y la violencia familiar. El enfoque dado es desde la educación, ya que se parte que entre otros muchos factores, se trata de un problema educativo concretamente referido a la gestión de las emociones. Se parte de la idea de que la violencia es el resultado de un conflicto no resuelto que ha ido creciendo a falta de herramientas o voluntad del sujeto para solucionarlo de forma pacífica. Por lo tanto, el punto de inicio a la hora de tratar este tema, debe comenzar por un acercamiento al conflicto en sí, a fin de evidenciar la raíz del problema para poder encontrar soluciones. Visto de esta forma, a lo largo de las siguientes páginas se muestra cómo muchas de las acciones o posicionamientos políticos, desde donde se dictan las leyes no son del todo acertados, ya que no parten de un análisis de la realidad, sino que lo que se pretende es atajar las consecuencias que éste genera. De ahí que el tema de la violencia de género hacia las mujeres y la violencia intrafamiliar hayan aumentado en las últimas décadas convirtiéndose en un problema mundial que preocupa a toda la sociedad, a excepción de los que ejercen la violencia o solo conocen el lenguaje violento. Para Rico

${ }^{13}$ Doctora por la Universidad Pablo de Olavide en el "Programa de Evaluación, Mejora y Calidad en la Educación Superior” de la Facultad de Ciencias Sociales, Sevilla, España. Profesora Contratado Doctor en la Facultad de Ciencias de la Educación de la Universidad de Cádiz, Cádiz, España. E-mail montse.vargas@uca.es 
(1996) la primera idea a defender, es que la violencia de género es una cuestión de derechos humanos, por lo que el tema alcanza una dimensión social y debe ser estudiado desde una perspectiva que permita la posibilidad de cambios culturales.

\section{Cuando la violencia es un conflicto no resuelto}

Al estudiar un concepto siempre se recomienda partir de la definición del mismo para especificar el punto de pArtida y facilitar así la comprensión, tanto del trabajo en sí como de las propuestas de intervención y tratamiento del objeto de estudio. La primera fuente consultada es la Real Academia Española (RAE) 2010 que da varios significados del vocablo "conflicto", que se define como: combate, lucha, pelea, enfrentamiento armado o problema, cuestión, materia de discusión. El conflicto desde un punto de vista psicológico hace referencias a "coexistencia de tendencias contradictorias en el individuo, capaces de generar angustia y trastornos neuróticos". Parece evidente que la tendencia es hacia una comprensión negativa del conflicto. La modificación de esta idea generalizada es quizás uno de los objetivos de aprendizaje propuestos para trabajar a lo largo de este tema.

Afortunadamente, esta visión negativa no es la que se asume desde el ámbito de la educación. Coincidiendo con autores como Medina (2011) se entiende el conflicto como una oportunidad para el desarrollo personal:

El conflicto es la confrontación de ideas, el encuentro entre dos posiciones que manejan criterios distintos frente a una misma problemática. Es una tensión en la relación social e interpersonal generada por una diferencia de intereses. Los encuentros entre dos o más personas siempre estarán cargados de puntos de divergencia, los cuales son espacios que implican salir de una zona de comodidad a una zona de riesgo, pero ofrecen una oportunidad excelente para la construcción de nuevos aprendizajes (p.13).

Entre las teorías con tendencia a centrar las causas del conflicto en motivaciones intrínsecas del sujeto, se puede destacar la idea sobre la transformación del conflicto aportada por Lederach (2009): 
La transformación del conflicto es una forma de visualizar y responder al ir y venir de los conflictos sociales como oportunidades que nos da la vida para crear procesos de cambio constructivo que reduzcan la violencia e incrementen la justicia en la interacción directa y en las estructuras sociales, y respondan a los problemas de la vida real en las relaciones humanas (p. 56).

Es interesante esta visión más amable del conflicto, al tiempo que se ve como necesario y bueno para el desarrollo y el crecimiento. Son muchos los autores, sobre todo desde el campo de la mediación que defienden todo un marco teórico que apoya esta idea. Se pretende por tanto, llegar a la idea de que las acciones deben buscar soluciones pacíficas fundamentadas sobre todo en la capacidad de diálogo, lo que supone un acercamiento a una verdadera trasformación del conflicto como medio de evitación de la violencia. Por otra parte el afrontar los conflictos de esta forma, supone visualizar y responder a ellos como parte del crecimiento y oportunidades de mejorar la sociedad. Se hace necesario un profundo análisis evaluador con intención de que las propuestas lanzadas a la sociedad, sean verdaderos cambios con carácter permanente y no acciones puntuales o políticamente correctas.

Estos cambios deben responder a 4 dimensiones: la dimensión individual, dimensión interpersonal e intrapersonal, la dimensión de la gobernanza o de las personas con capacidad de toma de decisiones y la dimensión social y de empoderamiento. Todas ellas estarían dentro de una macro dimensión que sería la estructural, que enfatiza en las causas profundas del conflicto, los referentes y modificaciones que se dan a nivel de las estructuras sociales, políticas y económicas. La dimensión cultural hace referencia a los cambios que se dan en los patrones de comportamiento grupal, afectando la identidad y la cultura en la forma en que se asumen los conflictos.

Una de las primeras cuestiones susceptible de ser cambiadas para afrontar el problema, es asumir que el conflicto en sí es inherente al ser humano, de ahí la importancia de entenderlo como parte de la vida y del desarrollo, no evitarlo o en el mayor de los casos, negarlo. El conflicto no resuelto, entre otras cuestiones, lleva a la formación de personas desde el resentimiento, la ira, la desconfianza y otros sentimientos no apropiados para mantener una relación saludable con los demás. De ahí, la insistencia en que detrás de 
una persona violenta hay una historia de vida sin resolver y emociones sin una correcta gestión. Para conseguir la mejora y trasformación del sujeto algunos aspectos fundamentales como la empatía, resiliencia, aceptación de las diferencias y, sobre todo, la capacidad de diálogo y negociación, además de la humildad, reflexión e introspección, son fundamentales para la resolución de conflictos y la formación de la persona.

Respecto a la violencia de género y violencia intrafamiliar entendidas como un conflicto no gestionado, se ha considerado que la conducta del maltratador está asociada a cuestiones de trastorno psíquico, así como a carencias personales de la infancia o problemas de conducta en general. En tiempos pasados, fueron estos motivos personales los que han tratado de justificar la violencia de género, pero hoy en día deben ser el reto a superar y llevarlas a una realidad que rompan con lo que señala Expósito y Moya (2005): "las teorías sociales y culturales, por su parte, abogan por la existencia de valores culturales que legitiman el control del hombre sobre la mujer" (p.20).

La cuestión de las diferencias según el género, así como la supuesta superioridad del hombre sobre la mujer que se ha dado por impregnación generacional. De Keijzer (1997) aclara la cuestión del género y su significado social:

Por género entiendo una serie de atributos y funciones que van más allá de lo biológico/reproductivo, construidos social y culturalmente y que son adjudicados a los sexos para justificar diferencias y relaciones de opresión entre los mismos. El género se interioriza a través de todo un trabajo de socialización, entendida como un complejo y detallado proceso cultural de incorporación de formas de representarse, valorar y actuar en mundo (p. 3).

Las dimensiones individuales, sociales y de estructura que pueda tener el conflicto, se identifican también entre las causas de la violencia de género, con las teorías del conflicto donde se parte de una cuestión de nivel personal que lleva a tener problemas en las relaciones interpersonales y finamente se refiere a una cuestión cultural o social. En ningún caso se pretende justificar cualquier tipo de violencia. Lo que se pretende es evidenciar la necesidad de conocer las causas o carencias a nivel emocional 
o psíquico que llevan a que una persona tenga una conducta agresiva y violenta hacia las mujeres en particular. Se hace necesario y urgente actuar desde el convencimiento de la necesidad de una trasformación de la sociedad y la erradicación total de la cuestión a través de la educación.

\section{Dimensión social de la violencia de género}

Respecto al estudio sobre la violencia de género y la violencia doméstica, si bien en muchas ocasiones la tendencia es la de igualar ambos términos, se entiende que son diferentes. Es importante una correcta identificación a fin de no caer en actuaciones erróneas y esfuerzos que no son orientados a dar respuestas acertadas. Para Abreu (2006) desde un punto de vista jurídico, el problema radica en una cuestión cultural y estructural lo expresa así:

El género se constituye así en el resultado de un proceso de construcción social mediante el que se adjudican simbólicamente las expectativas y valores que cada cultura atribuye a sus varones y mujeres. Fruto de ese aprendizaje cultural de signo machista, unos y otras exhiben los roles e identidades que le han sido asignados bajo la etiqueta del género. De ahí, la prepotencia de lo masculino y la subalternidad de lo femenino. Son los ingredientes esenciales de ese orden simbólico que define las relaciones de poder de los hombres sobre las mujeres, origen de la violencia de género (p. 2).

Son muchos los autores que coinciden en que la violencia de género se ha dado a lo largo de la historia por una cuestión de machismo en la que el hombre se empodera por entre otras, una cuestión económica. Quizás sea la incorporación de la mujer al mundo del trabajo y su independencia un factor importante que ha supuesto un aumento de la violencia doméstica, por lo que se podría tratar de un problema de sumisión asociada al género femenino, por una cuestión cultural. Autores como Soleto, Zafra Espinosa de los Monteros y otros (2015) coinciden en que se trata de una cuestión de estructura social:

El problema de la violencia de género es un problema estructural intrínseco de la sociedad, proveniente las sociedades y educaciones patriarcales, dónde el hombre por el hecho se serlo, se consideraba superior a la mujer, y por ende, esta debía respetarlo, cuidarlo y permitir cualquier situación que proviniera de él, incluso la dominación (p. 13). 
Aportaciones como la de Montero Hechavarría, y otros (2015) así como Cárdenas Callol (2011) coinciden en esta trasmisión cultural. Idea que comparte Abreu (2006) considerando que la violencia contra las mujeres es en clave cultural y no biológica. Montero Hechavarría (2015) señalan:

Hay una raíz cultural histórica primeramente, pues durante mucho tiempo la sociedad ha sido muy machista, el hombre ha creído que tiene el derecho primario a controlar, a disciplinar con severidad, incluso a abusar de la vida de la mujer y los hijos, lo cual ha sucedido bajo la apariencia de la función económica del hombre, quien es proveedor de la alimentación (párr.4).

Ante esta visión de la violencia de género como una cuestión de estructura social, autores como La Parra y Tortosa (2003) consideran que hay un tipo de violencia que es evidente, donde víctimas y agresor son fácilmente identificados, pero existe otra violencia que es invisible donde es difícil reconocer a los que la sufren. Se refiere el autor a una violencia estructural, ya que la causa está en los procesos socialmente estructurados y que se han dado siempre, sin más, en las distintas estructuras, incluida la familia. Es la ausencia de una violencia directa la que la hace permanente en la historia, convirtiéndose en una "normalidad" no por pertinencia, sino por frecuencia.

\section{Distintos tipos de violencia referida al género $y$ violencia familiar}

A nivel normativo se aprecia que el tema de la violencia de género ha llegado a tales niveles que ha tenido que ser debidamente legislado para poder demostrar su visibilidad a fin de dar protección a aquellas mujeres que la sufren. La Ley Orgánica 1/2004, de 28 de diciembre, de Medidas de Protección Integral contra la Violencia de Género, reconoce que en España las agresiones a las mujeres son de gran incidencia, a causa de lo cual muchas organizaciones de mujeres han luchado por crear una sensibilidad social y para que pase de ser un "delito invisible" a crear un rechazo colectivo que genere una alarma social:

Violencia que, como manifestación de la discriminación, la situación de desigualdad y las relaciones de poder de los hombres sobre las mujeres, se ejerce sobre estas por parte de quienes sean o hayan sido sus cónyuges 
o de quienes estén o hayan estado ligados a ellas por relaciones similares de afectividad, aun sin convivencia (Art. 1.1).

Una lectura detallada de este artículo de la ley citada, puede llevar a pensar que se está ante lo que pudiera ser un problema de discriminación positiva, ya que solo se considera la violencia de género del hombre hacia la mujer y no al contrario. Otros estudios evidencian que el maltrato de la mujer al hombre suele ser de carácter psicológico y no de agresión física, por cuestiones evidentes de fuerza, pero sí que existen. Entendemos que este debe ser uno de los retos de la educación: romper los mitos de que en la violencia de género el hombre "siempre" es culpable. Por otra parte, tampoco se consideran los distintos tipos de parejas que actualmente nos encontramos, como pueden ser las parejas homosexuales, donde la violencia también se da. Aportaciones como las de Gimeno Reinoso y Barrientos (2009) señalan que en EEUU las denuncias de malos tratos entre parejas homosexuales han llevado a la creación de un sistema de apoyo desde las instituciones de asuntos sociales. Esto respalda la idea de que la Ley antes mencionada no responde completamente a las necesidades sociales planteadas en relación a la violencia de género, ya que deja al descubierto lo que sería la violencia doméstica, al entender que solo se contemplan aquellas agresiones del hombre hacia la mujer y con necesidad de una convivencia actual o en el pasado. Se entiende por tanto que estos factores no cubren todo el problema de género en su totalidad, tal y como dice pretender la Ley. Por lo tanto, se abre un nuevo debate donde no solo hay que distinguir entre violencia de género y violencia doméstica, sino aceptar que hay otros tipos de violencia en las que no tienen necesariamente que darse en una convivencia.

Desde este posicionamiento, la educación debe mostrar que la violencia de género es aquella que se ejerce por la simple razón de ser de género diferente, siendo el género femenino el que con mayor frecuencia se sitúa en la posición de víctima, por cuestiones culturales, donde la mujer es considerara en muchos casos un objeto que puede ser poseído por el varón. Este tiempo de violencia no es exclusivo en el seno familiar o implicar una relación afectiva. Se puede dar en cualquier ámbito ya sea familiar, laboral o de relaciones sociales. El detonante de la violencia en este caso es el género; 
porque ser mujer. Por otra parte, la violencia intrafamiliar se debe entender como aquella que se da dentro del seno de la familia hacia cualquiera de sus miembros. Como vemos, las agresiones de los hijos hacia los padres o la que sufren los ancianos y los niños, no deben ser confundidos con la violencia de género ya que en este caso el motor o la causa es la vulnerabilidad respecto al otro. En aras a buscar soluciones, es importante hace esta distinción a fin de que las leyes las puedan contemplar y adoptar medidas para su erradicación. Medidas que deben partir del respeto, fomento del diálogo y aceptación del otro y de uno mismo. Para ello se necesita un sistema educativo centrado en el sujeto y no en los contenidos, que posibilite el autoconocimiento y que suponga un reto personal de mejora como persona.

Se entiende que la familia es el referente y primer agente socializador que debe asegurar el bienestar emocional y físico, entre otros, y donde se mezclan las relaciones que se puedan dar en toda organización. No obstante, a diferencia de estas, la familia tiene una connotación emocional y de amor que la hace más fuerte y vulnerable al mismo tiempo. Por ello, se hace fundamental la comunicación y formación paralela de la familia y su participación activa en la escuela.

\section{Detectar la violencia intrafamiliar y la violencia de género}

Como parte de la reflexión sobre el complejo tema de la violencia intrafamiliar y de género, se hace necesario abordar un tipo de violencia que según los autores es cada vez más frecuente en España. Las investigaciones hacen especial referencia a la violencia filo-paternal que han estudiado autores como Calvete, Orue, y González-Cabrera (2017) o Gámez-Guadix, Jaureguizar, Almendros, y Carrobles (2012) quieres concluyen que este tipo de violencia tiene una estrecha relación con el modelo de educación que se desarrolla en la familia, distinguiendo entre padres autoritarios, democráticos y permisivos. Obviamente en cada tipo de educación se ponen en juego o se trabajan distintas competencias que llevan a la formación del sujeto. Es sabido que el modelo de padres democráticos lleva al razonamiento, reflexión, responsabilidad y consecuencias de los actos y sobre todo al poder del diálogo y el entendimiento. Según señala los autores, la violencia de hijos a padres es algo que va en aumento: 
Las agresiones verbales y físicas de los hijos son cada vez más frecuentes y se dan en una franja de edad entre los 14 y 17 años, porcentaje que aumentaba considerablemente (hasta un 39,7\%) en la franja de edad de 17 años, llegando incluso a ser más alto que el porcentaje de expedientes abiertos por otro tipo de delitos (31,59\%) entre menores infractores (p. 590).

Según este estudio el problema se agrava por la falta de registros e investigaciones al respecto. Al parecer una vez más se identifica como un problema social que requiere de protocolos de detección temprana. Hay que tener en consideración que tanto los padres como madres, hermanos y demás miembros que componen el núcleo familiar están bajo un caso de violencia doméstica. Hay una gran dificultad a la hora de abordar este tema ya que supone el asumir que los progenitores son maltratados por un hijo, lo que implica cierto desconcierto y sentimiento de fracaso en el proceso de crianza. Por ello se propone que deberían ser los servicios sanitarios así como los profesionales de la educación, los que detecten el problema, les animen y acompañen en el proceso de denuncia. La actuación de asuntos sociales sería el siguiente escalón.

Otras investigaciones sugieren que la violencia filio-parental está relacionada con la historia previa de violencia en el seno familiar. En estudios como los realizados por Contreras y Del Carmen (2016) muestran unos resultados donde los menores que agredían a sus padres presentaban mayores niveles de exposición a la violencia en el hogar, lo que se relacionaba significativamente con la percepción social hostil de los adolescentes.

El poder de las relaciones dentro del núcleo familiar tiene una gran peculiaridad, que hace muy difícil detectar el maltrato antes de que llegue a ser considerado violencia. Nos referimos a que normalmente no deja huellas físicas o que el sujeto víctima de este maltrato, pueda llegar a identificar como tal lo que sobre él se está ejerciendo. Especialmente a las acciones negativas que algunos tipos de padres lleguen a ejercer sobre sus hijos de una forma sutil, muy difícil de apreciar. Se habla de padres narcisistas, tóxicos o a aquellos que tienen un Síndrome de Alienación Parental (SAP).

Se trata por tanto de una cuestión de cómo se ejerce el poder sobre el que, en principio, es más débil o vulnerable. En este caso se considera una 
relación destructiva, ya sea a nivel físico, psicológico o emocional, ejercida hacia los hijos e incluso hacia las personas mayores, tema que se trata en otro apartado. Otros autores consideran que: "La violencia, el maltrato, la injusticia son en cada ocasión únicos e igual de hirientes para las víctimas y todas ellas requieren cuidado y atención, así como todos los maltratadores o agresores merecen su correspondiente sanción pena" (Gimeno Reinoso y Barrientos Silva, 2009, p. 7). De ahí la necesidad de empezar por un cambio de mentalidad desde la educación, donde los valores de respeto, igualdad, equidad, bien común y sobre todo el valor de la vida, sean el hilo conductor para crear una sociedad democrática donde se respeten los valores fundamentales. En otra línea de actuación, son muchos los estudios que consideran que la violencia infligida por la pareja (IPV) y los trastornos mentales (MD) constituyen un importantes problemas de salud pública que afectan desproporcionadamente a las mujeres (Ruiz-Pérez, Rodríguez-Barranco, Cervilla, y Ricci-Cabello, 2018).

Como ya se señaló en el apartado anterior, la violencia doméstica se puede dar entre cualquier o hacia cualquier miembro de la familia. Montero Hechavarría, Delis Tabares, Ramírez Pérez, Milán Vázquez y Cárdenas Callol (2011) consideran que dentro de esta violencia se encuentra el maltrato infantil, maltrato físico, maltrato sexual, abandono físico, abuso y abandono emocional, niños testigos de violencia conyugal y maltrato a ancianos. Pero también se pueden incluir otras formas de violencia como el abandono emocional o afectivo y la negligencia en los cuidados, dado por la falta de protección y cuidados físicos de los miembros de la familia que lo requieran, la falta de respuesta a las necesidades de contacto afectivo y estimulación cognitiva, falta de atención, descuido (Almenares Aleaga, Louro Bernal, y Ortiz Gómez, 1999).

Merece una especial atención a este último tipo de violencia doméstica ya que los datos aportados por Sanmartín, Torner, Martí, Izquierdo, Solé y Torrellas (2001), muestran que la prevalencia estimada de malos tratos a ancianos oscila en un 3-10\% según varios estudios y su incidencia ha aumentado un $150 \%$ en 10 años. Consideran estos autores que se podrían estar ante lo que denominan fenómeno iceberg donde cinco de cada seis casos quedan sin notificar. En el estudio se identifica la violencia doméstica hacia las personas mayores donde se engloba abusos de tipo físico, psicoló- 
gico o emocional, sexual, económico o financiero, negligencia, abandono y autonegligencia (2001). El estudio dio como resultado que "los autores del posible maltrato fueron hijos en un $57 \%$ de los casos, yernos/nueras en el $23 \%$, cónyuges en un $8 \%$ y otros en el resto. De los posibles causantes de maltrato, un $19 \%$ presentaba toxicomanías y el $15 \%$ problemas psiquiátricos" (2001, p.333). Asimismo, se abre una vía de atención y detención de la violencia doméstica que según los autores debe ser detectada en los servicios sanitarios de atención básica, una cuestión que hasta ahora ha sido infravalorada.

Parece que se evidencia la necesidad de abordar el tema de violencia tanto de género como intrafamiliar desde el campo sanitario. Son muchos los autores que coinciden en esta idea y cada vez hay más estudios y personal sanitario implicado, tal y como señalan Calvo González y Camacho Bejarano (2014).

Por su parte, la ONU (2017) considera que "la violencia contra la mujer, especialmente la ejercida por su pareja y la violencia sexual, constituye un grave problema de salud pública y una violación de los derechos humanos de las mujeres". (2017, párr. 1). Se apuesta por la necesidad de una sensibilización de la sociedad en general y sobre todo por una formación en el personal médico sanitario a fin de poder detectar los casos de violencia de género o familiar. Los datos aportados por el estudio de los autores anteriormente citados señalan que "las mujeres víctimas de violencia acuden más a los centros de salud y presentan mayor estrés y problemas psicológicos, síntomas concomitantes y mayor uso de antidepresivos (...) además de desarrollar más problemas cardíacos y dolores musculo-esqueléticos en el cuello y en la espalda y otras manifestaciones sistémicas" (2017, p.431). La cuestión es difícil de abordar ya que aparecen barreras organizativas, como señalan los autores, tales como la falta de protocolos de detección y asistencia, la alta presión asistencial, y la falta de profesionales cualificados.

\section{Hacia una solución rompiendo mitos y estereotipos}

Uno de los problemas que tiene peor solución es la cuestión del aprendizaje por impregnación generacional. Investigaciones como las de Luz Magdalena (2005) demuestran que los jóvenes que se han criado en entornos de violencia familiar tienden a reproducir estas actuaciones cuando 
forman sus propias familias. Desde la educación, se asume que los menores aprenden por imitación, eso es obvio y aceptado, pero no es acertado quedarse ahí. Algunos autores cuestionan esta afirmación y personalmente se considera que si partimos de esta premisa; padre maltratador- hijo maltratador, será difícil o casi imposible encontrar soluciones para el cambio. Por otra parte, los educadores deben confiar en el poder de la educación y su capacidad para generar cambios.

Delgado-Álvarez, Sánchez y Fernández-Dávila (2012) hacen una interesante aportación sobre los estereotipos de masculinidad o feminidad, así como al de asociar la fuerza y la responsabilidad del mantenimiento económico del hogar al varón o el de las mujeres como cuidadoras y emocionales. Son algunos de los estereotipos que puedan estar detrás de lo que denomina el ciclo de la violencia de género, caracterizado por la permanencia de la víctima en la convivencia con el maltratador, en una sucesión alternante de episodios violentos seguidos de episodios de reconciliación, que progresan en una espiral de violencia cada vez mayor.

En su estudio también se plantea la trasmisión que hacen los medios sobre las características de ambos sexos, encontrando una asociación entre la violencia doméstica y los atributos de género masculino. Se percibe a los hombres como abusivos y personas violentas, mientras que las mujeres son vistas como pasivas y se aprecia la noción de coraje como la principal característica necesaria para romper el ciclo de la violencia.

Los mitos sobre la marginalidad o que la violencia de género solo ocurre en países subdesarrollados o en familias/personas con problemas (pocos recursos, desempleo...). Son cuestiones que se deben ir cambiando si realmente se busca una trasformación social en la que se hable de Derechos Humanos, respeto, equidad de género y sostenibilidad social. Autores como Bosch-Fiol y Ferrer-Pérez (2012) hablan de la necesidad de romper estos mitos establecidos socialmente. Hoy en día, tanto la violencia de género como la violencia intrafamiliar o doméstica, lamentablemente afecta a todos las clases sociales. Quizás dependa del nivel de dependencia psicológica, emocional y económica que la víctima tenga con su agresor, lo que le permita salir de una situación de violencia. No todas las mujeres u hombres llegado el caso, pueden tener la posibilidad, ya sea por cuestiones de 
economía o cuestiones de autoestima de poder denunciar o plantearse otro tipo de vida. La manutención de los hijos sería entre otras una cuestión a resolver. En este caso Vives-Cases, y otros (2009) hablan de una "población vulnerable" donde se incluyen subgrupos de población que tienen características similares siendo precisamente su posición en la estructura social lo que limita o imposibilita la determinación al cambio.

Respecto al mito del maltratador, es casi asumido por la mayoría, que los hombres que maltratan a su pareja (o ex pareja) han sido a su vez, personas maltratadas por sus padres, o han sido testigos de maltrato en su familia de origen (hipótesis de la transmisión intergeneracional de la violencia de género) Bosch-Fiol y Ferrer-Pérez (2012). Asimismo, el autor rebate el mito de que los hombres que maltratan a su pareja (o ex pareja) son enfermos mentales o que consumen/abusan de alcohol y/o drogas. La interesante reflexión de Careaga Castrillo (2010) lleva a rechazar este mito, ya que tal y como ejemplifica la autora, no se entiende que estas personas violentas agredan solo a las mujeres, si realmente están bajo los efectos del alcohol o con enfermedad mental ¿por qué no son agresivos con todos? No se puede justificar un maltrato considerando al agresor víctima de su propia historia y al mismo tiempo hacer sentir a la víctima culpable por no entender sus limitaciones o condicionantes.

Si bien es cierto, como ya se ha señalado anteriormente, que los niños aprenden por imitación, no se debe condicionar y caer en una profecía auto cumplidora al pensar que de padres violentos necesariamente tienen que resultar hijos violentos, $\mathrm{Si}$ se acepta esto, se estaría negando el poder de la educación para trasformar realidades sociales. Los estudios realizados por Peña, Pérez-de-Guzmán y Maurandi (2015) concluyen en que:

Existe unanimidad sobre la necesidad de recibir formación en educación para la igualdad, prevención y detección de la violencia de género, y en detección precoz de la violencia en el ámbito familiar, dado que la consideran fundamental para poder desempeñar su futura labor profesional (p. 61).

Pero quizás el mito que más llama la atención es el de el motivo son los celos y la repetida frase socialmente asumida por lo que justifican cualquier 
tipo de violencia de que "Si las mujeres padecen violencia de género algo habrán hecho para provocarla”. En este sentido, en la Declaración sobre la eliminación de la violencia contra la mujer (Resolución de la Asamblea General 48/104, ONU, 1993) se colocó la violencia contra las mujeres en el marco de los Derechos Humanos, explicitando que las mujeres tienen derecho a disfrutar de los derechos y libertades fundamentales y a que éstos sean protegidos, y que las diferentes formas de violencia contra las mujeres son violaciones de los derechos humanos (Heyzer 2000). La Declaración y Plataforma de Acción de Beijing (ONU 1995) subrayó que "la violencia contra la mujer es a la vez una violación de los derechos humanos de las mujeres y un obstáculo para el pleno disfrute de todos los derechos humanos por parte de las mujeres" (p.550), refiriéndose a conceptos tales como microviolencias o micromachismos donde se incluyen o justifican los delitos propios al género.

Si bien hay unos estereotipos relacionados a los maltratadores que se deben cambiar, también los hay en relación a las víctimas. Estudios como los de Musach, Alonso, Guitart, Castañe y Esquius (2010) demuestran que trastornos como pueden ser los psicológicos de las mujeres maltratadas, surgen como consecuencia de la violencia domestica (VD) siendo el maltrato el motivo por el que se genera. El consumo de alcohol, abuso de las drogas o el nivel cultural de la víctima de VD y no se encontró relación entre la VD y el análisis de los hábitos tóxicos. El estudio coincide con otros en que hay una negación del problema donde las víctimas no reconocen estar bajo una situación de violencia doméstica y llegan a identificar su relación como normal

La Ley Orgánica 1/2004, de 28 de diciembre, de Medidas de Protección Integral contra la Violencia de Género, en su Artículo 6 sobre Fomento de la igualdad, establece que: "con el fin de garantizar la efectiva igualdad entre hombres y mujeres, las administraciones educativas velarán para que en todos los materiales educativos se eliminen los estereotipos sexistas o discriminatorios y para que fomenten el igual valor de hombres y mujeres" Se desprende que en la mayoría de los casos no alcanza más que a ser una declaración de intenciones. El hecho de que se apueste y se parta de una idea de igualdad, ya se orienta erróneamente. Hombres y mujeres son diferentes, sienten diferentes, piensan diferente y se realizan de dife- 
rente manera. No se trata de igualdad, sino más bien de equidad. De una forma u otra, se impone la necesidad de aceptar que la mujer tiene un rol de "cuidadora social" difícil de erradicar en las mentes de todos. Pero esto no tiene que suponer que por el hecho de ser mujer necesariamente tenga que cumplir ese rol. El ignorar este punto de vista donde se apuesta por hablar de equidad y no solo de igualdad, sin asumir la diferencia como la realidad, lleva a que las leyes, como puede ser las de conciliación familiar, en muchos casos sigan perjudicando a la mujer o en otros casos son los hombres los que salen seriamente desprotegidos legalmente, por el hecho pertenecer al género varón.

En muchas separaciones matrimoniales, son los hombres los que deben abandonar la vivienda, dejar a sus hijos y hacer una aportación económica mensual. Muchos pueden pensar que esto es justo, pero esta medida no está exenta de polémica. Obviamente lo que procede es que se haga cargo de sus responsabilidades como padre, pero que la ley no atienda a los casos particulares y que sea el género el que determine la vida de todos, es lo que se debe llevar a la reflexión. Por lo tanto, sería necesario empezar por hablar de equidad de género, para hablar de condiciones para igualdad. Lamentablemente parece que ya hay muchas voces que detectan que la cuestión del género se está polarizando y lejos de ir en la dirección de una sociedad donde se respeten los derechos de todos, vamos en ocasiones hacia un empoderamiento inadecuado de la mujer, tendiendo hacia una discriminación positiva y que puede estar convirtiendo al hombre en victima por el hecho de ser hombre. Posiblemente esté surgiendo el nacimiento de una nueva discriminación que puede llevar a otro tipo de violencia, ya que otra vez el motor es el pertenecer a un género determinado.

Afortunadamente este planteamiento está comenzando a ser tratado en la literatura científica. Trabajos como los de Trujano, Martínez y Camacho (2010) consideran que, "las leyes de protección a las víctimas deberían ser lo suficientemente incluyentes para todos, especialmente si consideramos temas pendientes como las nuevas relaciones que se están gestando de manera cada vez más abierta en las sociedades actuales" (p.340). Compartiendo esta idea se asume que el hablar de la violencia de la mujer contra el varón no es políticamente correcto y nuevamente se estaría haciendo una diferencia por cuestiones de género, donde como ya se comentó, el rol de 
violento se atribuye por defecto, tradición cultural y social al hombre. Los datos que portan los autores en su estudio, son los publicados en el Anuario 2005 Ministerio del Interior y señalan:

En España, en el año 2000 fueron asesinados 44 hombres por sus esposas; 16 más en el 2002. En el 2005 la cifra fue de quince. Pero si contamos a todos los varones muertos en el ámbito familiar por cónyuge, ex cónyuge, novia, compañera sentimental, hijo/a, padre/madre, o pariente, tenemos que suman 56 , representando el $48.2 \%$ de la cifra total (2010, p. 341).

Se aprecia una similitud en el tipo de maltrato y que al igual que el caso de violencia hacia la mujer, se sigue el ya conocido ciclo de la violencia que se inicia con una etapa de acumulación de tensión, segunda etapa de crisis o descarga de la violencia y una última etapa que se conoce como luna de miel, en la que se dan los arrepentimientos, victimización del agresor, sentimiento de culpa de la víctima y promesas que se romperán cuando se vuelva a iniciar el ciclo.

En el caso de la violencia de género hacia las mujeres, los estudios (Calvo González y Camacho Bejarano, 2014) evidenciaban que gran parte del problema es que se están ante una "infradetección” ya que solo se diagnostica un pequeño porcentaje de éstos y con una demora de entre 6 y 10 años desde que se inician las primeras agresiones, siendo un motivo de preocupación por las consecuencias que para la víctima y para los hijos tiene una situación de violencia mantenida durante muchos años. Se debe pensar que en caso de los varones esta situación puede aún ser más complicada ya que están fuertemente condicionados por el rol social. El caso de los hombres víctima, algunos estudios apuntan hacia un inicio del mal trato desde el periodo de noviazgo.

\section{La educación de las nuevas generaciones. Primer paso hacia una solución.}

Tanto los datos cualitativos como cuantitativos, así como estudios sobre factores que inciden en el problema de la violencia de género y violencia intrafamiliar, son suficientes para poder establecer la necesidad de crear políticas educativas, de detección y apoyo a las familias que tal y como 
propone Rey (2002), brindan la posibilidad de crear campañas de prevención primaria y secundaria, así como lo relacionado al maltrato infantil y delincuencia juvenil.

Una de las consecuencias de vivir en un ambiente familiar de violencia es que afecta sobre todo a los menores y condiciona su futuro. A lo largo de este capítulo se ha evidenciado que las consecuencias no se materializan solo en el momento de sufrir esta violencia, sino que pueden ser significativas y condicionantes para toda su vida de adulto. Estudios como los de Preto y Moreira (2012) hablan de un bajo rendimiento académico, ya que en este proceso entran en juego los pensamientos, sentimientos y las expectativas de logro ante la obtención de una meta, por lo que se concluye que la violencia doméstica contra las mujeres afecta negativamente al desarrollo integral de la persona.

Los datos que ofrece Ruiz (2014) respecto al papel de las redes sociales en los temas de violencia de género, muestran que la intervención en el sistema educativo es una cuestión de urgencia. Ante la pregunta ¿crees que si una persona es celos es porque te quiere? Los alumnos de primero de la ESO responden un $86 \%$ afirmativamente las chicas y un $73 \%$ los chicos. Afortunadamente el valor baja en función a la edad, llegando a unos valores de del $40 \%$ los chicos de $2^{\circ}$ de bachillerato y un 13\% las chicas del mismo curso. Como ya se dijo, uno de los mitos de la violencia de género era que se actuaba por cuestiones de celos. En relación a que si los sujetos encuestados, justifican la violencia, la autora nos muestras unos datos en los que el $100 \%$ de los alumnos varones de segundo de bachillerato responden negativamente, mientras que las chicas del mismo curso, justifican la violencia en un 20\%. En su estudio, una de las hipótesis de partida era demostrar que "través del uso de las redes sociales podemos comprobar cómo el discurso del amor romántico justifica mecanismos de control de la pareja” (p.285).

Se asume que la adolescencia se considera como un periodo preparatorio para la edad adulta. Es donde se forja la identidad personal al tiempo que se refuerzan o rompen las relaciones personales y sociales que el sujeto ha mantenido hasta entonces. Es en la adolescencia cuando el sujeto toma conciencia de sí mismo, tratando de imponer su yo más exacerbado. Será la 
institución educativa la que tenga que estar dotada de buenos profesionales capaces de detectar, atender y frenar cualquier atisbo hacia la violencia que se pueda dar. Partiendo de que actualmente el desarrollo de las personas se da en una sociedad eminentemente violenta y que las faltas de respeto y abandono de los valores son en muchos casos una realidad, hoy más que nunca, la intervención desde la educación se hace necesaria y fundamental para mitigar los efectos nocivos que esa sociedad dañada pueda hacer en el proceso de formación de la persona, lo que se conoce como educación informal.

Finalmente, hay que destacar el papel que juega la familia. Como primera institución socializadora del sujeto, por ello debe centrarse en dotar de las herramientas y competencias personales que le permitan resolver conflictos. Tanto a nivel personal, como los que se puedan generar con las personas con las que interactúa. El panorama familiar en general puede no resultar muy alentador y viéndolo de esta forma, es fácil pensar que el conflicto está garantizado, es más, lo extraño sería que no existiera. Por ello, una de las claves es el diálogo como herramienta para el entendimiento así como dotar al sujeto que aprende, de competencias en la resolución de conflictos y gestión de las emociones. Las metodologías de formación deben centrarse en posibilitar que el sujeto se conozca así mismo, descubriendo sus limitaciones y potencialidades. El continuar con un sistema educativo basado en la instrucción supone no solo un alejamiento de las soluciones, sino que fomenta la existencia de cualquier tipo de violencia que se genere como consecuencia de un conflicto no resuelto por falta de herramientas para el diálogo.

La educación, aparte de ser una cuestión de sutilezas es una cuestión sobre todo de coherencia. 


\section{Referencias bibliográficas}

Abreu, M. L. M. (2006). La violencia de género: Entre el concepto jurídico y la realidad social. Revista Electrónica de Ciencia Penal y Criminología, (8), 2.

Almenares Aleaga, M., Louro Bernal, I., y Ortiz Gómez, M. T. (1999). Comportamiento de la violencia intrafamiliar. Revista cubana de Medicina general Integral, 15, 285-292.

Bosch-Fiol, E., y A. Ferrer-Pérez, V. (2012). Nuevo mapa de los mitos sobre la violencia de género en el siglo XXI. Psicothema, 24 (4), 548-554.

Calvete, E., y Orue, I., y González-Cabrera, J. (2017). Violencia filio parental: comparando lo que informan los adolescentes y sus progenitores. Revista de Psicología Clínica con Niños y Adolescentes, 4 (1), 9-15.

Calvo González, Germán, y Camacho Bejarano, Rafaela. (2014). Gender violence: trends, impact and keys for approach. Enfermeria Global, 13(33), 424-439. Recuperado el 25 de marzo de 2018 de http://scielo.isciii.es/ scielo.php?script=sci_Arttextypid=S1695-61412014000100022ylng=es ytlng=en.

Careaga Castrillo, P (2010). Para Erradicar La Violencia Contra Las Mujeres (XII Seminario Duque de Ahumada sobre Violencia de Género) disponible en http://portal.uned.es/pls/portal/docs/PAGE/UNED_ MAIN/LAUNIVERSIDAD/UBICACIONES/06/DUQUE_ AHUMADA/17/PONENCIA_PILAR_CAREAGA.PDF

Contreras, L., y del Carmen Cano, M. (2016). Child-to-parent violence: The role of exposure to violence and its relationship to social-cognitive processing. The European Journal of Psychology Applied to Legal Context, $8(2), 43-50$.

De Keijzer, B. (1997). El varón como factor de riesgo: masculinidad, salud mental y salud reproductiva. Tuñón E. Coordinadora. Género y salud en el sureste de México. Villa Hermosa: ECOSUR/UJAD, 67-81. 
Delgado-Álvarez, M. C., Sánchez, G. M. C. y Fernández-Dávila, J. P. A. (2012). Atributos y estereotipos de género asociados al ciclo de la violencia contra la mujer. Universitas Psychologica, 11 (3), 769-777.

España. (2005). Ley Orgánica 1/2004, de 28 de diciembre, de Medidas de Protección Integral contra la Violencia de Género. La Ley.

Española, R. R. A. (2010). Ortografía de la lengua española. Espasa.

Expósito, F., y Moya, M. (2005). Violencia de género. Aplicando la psicología social, 201-227.

Gámez-Guadix, M., Jaureguizar, J., Almendros, C., y Carrobles, J. A. (2012). Estilos de socialización familiar y violencia de hijos a padres en población española. Psicología conductual, 20(3), 585.

Gimeno Reinoso, Beatriz, y Barrientos Silva, Violeta. (2009). Violencia de género versus violencia doméstica: La importancia de la especificidad. Revista Venezolana de Estudios de la Mujer, 14(32), 27-42. Recuperado el 25 de marzo de 2018, de http://www.scielo.org.ve/scielo.php?script=sci_ Arttextypid=S1316-37012009000100004ylng=esytlng=en.

Lederach, J. P. (2009). El pequeño libro de transformación de conflictos. Bogotá, Iglesia Cristiana Menonita de Colombia. Centro Cristiano para la Justicia, Paz y Acción No Violenta. Bogotá.

Luz Magdalena, S. B. (2005). Transmisión intergeneracional de la violencia intrafamiliar: evidencia para las familias colombianas. Revista desarrollo y sociedad, (56), 285-337.

Medina, F. A. R. (2011). La convivencia desde la diversidad. Universidad Nacional de Colombia.

Mujeres, O. N. U. (1995). Declaración y Plataforma de acción de Beijing. Declaración política y documento resultado de Beijing, 5 .

Montero Hechavarría, E., Delis Tabares, M. T., Ramírez Pérez, R., Milán Vázquez, A. L., y Cárdenas Callol, R. (2011). Realidades de la violencia familiar en el mundo contemporáneo. Medisan, 15(4), 515-525. 
Musach, I. S., Alonso, A. R. H., GuitArt, R. R., Castañe, G. L., y Esquius, N. P. (2010). Violencia doméstica: preguntar para detectar. Atención primaria, 42(2), 70-77.

Peña, E. B., Pérez-de-Guzmán, V., y Maurandi, A. (2015). Formación en violencia de género en el Grado de Educación Social de las universidades españolas. Bordón. Revista de pedagogía, 67(3), 51-66.

Preto, M., y Moreira, P. A. (2012). Auto-regulação da aprendizagem em crianças e adolescentes filhos de vítimas de violência doméstica contra mulheres. Psicologia: Reflexão e Crítica, 25(4).

Rey, C. (2002). Rasgos sociodemográficos e historia de maltrato en la familia de origen, de un grupo de hombres que han ejercido violencia hacia su pareja y de un grupo de mujeres víctimas de este tipo de violencia. Revista Colombiana de Psicología, (11), 81-90.

Rico, M. N. (1996). Violencia de género: un problema de derechos humanos. UN. CEPAL. Unidad Mujer y Desarrollo. Serie Mujer y Desarrollo. No 16 44p. Recuperado de https://repositorio.cepal.org/ handle/11362/5855

Ruiz, M. Á. B. (2014). Implicaciones del uso de las redes sociales en el aumento de la violencia de género en adolescentes. Comunicación y Medios, (30), pág. 124.

Ruiz-Pérez, I., Rodríguez-Barranco, M., Cervilla, J. A., y Ricci-Cabello, I. (2018). Intimate pArtner violence and mental disorders: Co-occurrence and gender differences in a large cross-sectional population based study in Spain. Journal of affective disorders, 229, 69-78.

Sanmartín, A. R., Torner, J. A., MArtí, N. P., Izquierdo, P. D., Solé, M. C., y Torrellas, N. R. (2001). Violencia doméstica: prevalencia de sospecha de maltrato a ancianos. Atención Primaria, 27(5), 331-334.

Soleto Muñoz, H., Zafra Espinosa de los Monteros, R., Carmona, M. Á., Hernández. Moura, B., Ruiz López, C., Arias, F., y Ochaita Calvo, A. (2015). Violencia de género: tratamiento y prevención. Dykinson. 
Trujano, P., y Martínez, A., y Camacho, S. (2010). Varones víctimas de violencia doméstica: un estudio exploratorio acerca de su percepción y aceptación. Diversitas: Perspectivas en Psicología, 6 (2), 339-354.

Vives-Cases, C., Alvarez-Dardet, C., Gil-González, D., TorrubianoDomínguez, J., Rohlfs, I., y Escribà-Agüir, V. (2009). Perfil sociodemográfico de las mujeres afectadas por violencia del compañero íntimo en España. Gaceta Sanitaria, 23(5), 410-414. 